\title{
Eficacia de un programa de formación en competencias digitales aplicado a estudiantes del grado de magisterio en educación primaria basado en el modelo Affective elearning+
}

Effectiveness of a program of training in digital competences conducted with Primary Education Degree students on the affective e-learning+ model

\author{
José Antonio Ortega Carrillo*1 \\ jaorte@ugr.es \\ Lina María Rendón López** \\ lina.rendonlo@amigo.edu.co \\ Juan Antonio Fuentes Esparrell*** \\ fuentese@ugr.es \\ Álvaro Ortega Maldonado**** \\ alvaroortegamaldonado@gmail.com \\ * Universidad de Granada, España \\ ** Universidad Católica Luis Amigó, Medellín, Colombia \\ ***Universidad de Granada, España \\ ****ESCO-Universidad de Gales, España
}

\section{Resumen:}

INTRODUCCIÓN. El presente estudio analiza las diferencias estadísticamente significativas existentes en el grado de dominio de la competencia digital, contemplada desde 4 dimensiones y criterios de desempeño (navegación y resolución de problemas;

\begin{abstract}
:
INTRODUCTION. This study explores statistically significant differences in the degree of mastery of digital competence, contemplated from 4 dimensions and performance criteria (navigation and resolution of problems, legal provisions, co-
\end{abstract}

1 Dirección para correspondencia (correspondence address):

José Antonio Ortega Carrillo. Universidad de Granada. Departamento de Didáctica y Organización Escolar. Facultad de Ciencias de la Educación. Campus Universitario de Cartuja, s/n. 18071 Granada (España). 
Eficacia de un programa de formación en competencias digitales aplicado a estudiantes del grado de magisterio en educación primaria basado en el modelo Affective elearning+ josé Antonio Ortega Carrillo, lina María Rendón lópez, Juan Antonio Fuentes Esparrell y Álvaro Ortega Maldonado

disposiciones legales, derechos de autor y licencias; desarrollo y creación de contenido e interacción y gestión de la identidad digital), existente entre los estudiantes del grupo que participó en un programa de formación online basado en el modelo Affective eLearning+, y aquellos que actuaron de control. El plan formativo evaluado se llevó a cabo durante el desarrollo del proyecto I+D+i denominado Evaluación y desarrollo de dos competencias genéricas en estudiantes de primer año del grado de maestro en educación primaria, que finalizaron algo más de un centenar de estudiantes del Grado de Magisterio de Educación Primaria de las Facultades de Educación de la Universidad de Oviedo, la Universidad de Jaén y en el Centro Universitario SAFA (adscrito a la Universidad de Jaén). MÉTODO. Se aplicó un cuestionario de creación propia pretest-postest tanto al grupo experimental como control y se analizaron los resultados con las pruebas estadísticas de Kolmogorov-Smirnov, Levene y t de Student. RESULTADOS. Las diferencias significativas obtenidas tras aplicar el programa entre los grupos experimental y control presentan un balance claramente favorable en 9 de los 13 desempeños competenciales trabajados es decir del $70 \%$ de las competencias evaluadas. DISCUSIÓN. Ello subraya que el modelo Affective eLearnig+ es eficaz para desarrollar competencias profesionales digitales en un clima de bienestar y satisfacción en las personas a las que se aplica.

\section{Palabras clave:}

Affective eLearning+; Aprendizaje electrónico; competencia digital; educación superior; formación del profesorado; evaluación de programas; diseño cuasi experimental. pyrights and licenses, development and creation of content and interaction and management of digital identity) in a group of students that participated in an online training program based on the Affective e-Learning+ model, and those who acted as control. The training plan was carried out during the development of the $\mathrm{R}+\mathrm{D}$ $+\mathrm{i}$ project called Evaluation and development of two generic competences in first year Primary Education Degree students conducted at the Faculties of Education at the University of Oviedo, the University of Jaén and the SAFA University Center (attached to the University of Jaén). Over 100 students participated in the study. METHOD. A pretest-post-test self-created questionnaire was applied to both the experimental and control groups and the results were analyzed with the Kolmogorov-Smirnov, Levene and t-Student statistical tests. RESULTS. Statistically significant differences were obtained in favor of the experimental group for 9 of the 13 competences analyzed, that is, $70 \%$ of the evaluated competences. DISCUSSION. This emphasizes that the Affective eLearning+ model is effective in developing digital professional skills as long as there is a climate of well-being and satisfaction in the classrooms.

\section{Keywords:}

Affective e-Learning+; electronic Learning; competence; higher education; program evaluation; teacher education; quasiexperimental design.

\section{Résumé:}

INTRODUCTION. Cette étude analyse les différences statistiquement significatives dans le degré de maîtrise de la compétence numérique, vues à partir de 4 dimensions et critères de performance (navigation et résolution de problèmes; dispositions légales, droits d'auteur et licences; développement et création de contenu et interaction et gestion de I'identité numérique), existant entre les étudiants du groupe ayant participé à un programme de formation en ligne basé sur le modèle Affective eLearning +, et ceux qui ont joué le rôle de contrôle. Le plan de formation évalué a été réalisé lors du développement du projet $\mathrm{R}+\mathrm{D}+\mathrm{i}$ appelé Evaluation et développement de deux compétences génériques chez les élèves de première année du diplôme d'enseignant de l'enseignement primaire, 
Eficacia de un programa de formación en competencias digitales aplicado a estudiantes del grado de magisterio en educación primaria basado en el modelo Affective elearning+ josé Antonio Ortega Carrillo, lina María Rendón lópez, Juan Antonio Fuentes Esparrell y

qu'ont accompli un peu plus d'une centaine d'élèves du diplôme de l'enseignement primaire des facultés d'éducation de l'Université d'Oviedo, de l'Université de Jaén et du Centre universitaire SAFA (rattaché à I'Université de Jaén). MÉTHODE. Un questionnaire prétest-post-test auto-créé a été appliqué aux groupes expérimentaux et témoins, et les résultats ont été analysés avec les tests t de Kolmogorov-Smirnov, Levene et Student. RÉSULTATS. Les différences significatives obtenues après application du programme entre les groupes expérimental et témoin présentent un équilibre clairement favorable dans 9 des 13 compétences exercées, soit 70\% des compétences évaluées. DISCUSSION. Cela souligne que le modèle Affective eLearnig + est efficace pour développer les compétences professionnelles numériques dans un climat de bien-être et de satisfaction des personnes auxquelles il s'applique.

\section{Mots-clés:}

Affective eLearning+; e-learning; compétence numérique; enseignement supérieur; formation des enseignants; évaluation de programmes; conception quasi-expérimentale.

Fecha de recepción: 14-06-2020

Fecha de aceptación: 16-09-2020

\section{Introducción}

\section{Fundamentación teórica}

\section{Los precursores de la computación afectiva como base del modelo Affective eLearning}

Aunque el término computación afectiva fue acuñado por Picard (1997) para describir métodos informáticos relacionados, derivados o deliberadamente diseñados para influir en las emociones, son escasas las publicaciones existentes sobre Affective eLearning siendo las primeras de naturaleza empírica las publicadas entre los años 2007 y 2009.

La primera de ellas es el trabajo de Shen, Leon, Callaghan, \& Shen (2007) titulada "Exploratory Research on an Affective e-Learning Model".

La segunda, es igualmente de este mismo equipo de autores Shen, Callaghan, \& Shen (2008) y se titula "Afective e-Learning in residential and pervasive computing environments".

La tercera aparece un año después firmada por este equipo Shen, Wang, \& Shen (2009) y lleva por título "Affective e-Learning: Using "Emotional". Data to Improve Learning in Pervasive Learning Environment. Educational".

El primer artículo surge de un proyecto de investigación interuniversitario realizado entre el Centro de Estilos de Vida Digital de la Universidad de Essex, en el Reino Unido (http://digital-lifestyles.essex.ac.uk) y el 
Eficacia de un programa de formación en competencias digitales aplicado a estudiantes del grado de magisterio en educación primaria basado en el modelo Affective elearning+ josé Antonio Ortega Carrillo, lina María Rendón lópez, Juan Antonio Fuentes Esparrell y Álvaro Ortega Maldonado

Centro de e-Learning de la Universidad de Shanghai Jiaotong en China (www.dlc.sjtu.edu.cn). Explora cómo evoluciona la emoción durante el proceso de aprendizaje en línea para desarrollar sistemas de aprendizaje que reconozcan y respondan a los flujos de emociones del estudiantado. Construyeron un prototipo experimental de sistema de aprendizaje ajustado a las emociones expresadas, estudiando la relación existente entre emoción y aprendizaje. Usando el modelo afectivo de Russell encontrando hallazgos de interés, subrayando que el espacio emocional de los participantes fue compatible con dicho modelo. Los datos experimentales obtenidos también revelan la existencia de algunos bucles de aprendizaje que, hasta cierto punto, validan el modelo de Kort, hasta la fecha no validado en eLearning.

El segundo artículo examina cómo los modelos de computación afectiva emergentes pueden mejorar el aprendizaje electrónico. Se describen dos entornos digitales que desde una visión holística y conectada de la realidad adaptables al contexto presente y futuro de las necesidades del estudiantado en línea.

Tras ello, se explica la investigación que explora cómo evolucionan las emociones durante el proceso de aprendizaje, y cómo se puede aprovechar la retroalimentación emocional desde una concepción de aprendizaje electrónico adaptativo, con la finalidad de mejorar el rendimiento.

Los resultados reconocen cuatro tipos de emociones surgidas en el aprendizaje en línea a partir de las señales fisiológicas analizadas con una tasa de precisión de $86.5 \%$. Sus autores subrayan es el primer estudio publicado en el campo de la detección de emociones a partir de datos recopilados en sesiones de eLearning. Narran hallazgos sobre la evolución de las emociones durante el aprendizaje, siendo un avance en el objetivo de validar el modelo de espiral de aprendizaje de Kort en eLearning.

En el tercer artículo de la serie estos autores abordan el análisis global de los resultados obtenidos en su investigación referida a las tecnologías de detección de emociones a partir de señales biofísicas. En el mismo se propone y experimenta un modelo de e-Learning afectivo, con estudiantes que aprendieron usando la plataforma de e-Learning de la Universidad de Shanghai.

Esta fase de la investigación se inspiró tanto en el modelo de afecto circunflejo de Russell (1980) como en el modelo de espiral de aprendizaje de Kort (Kort, Reilly, \& Picard, 2001). 
Eficacia de un programa de formación en competencias digitales aplicado a estudiantes del grado de magisterio en educación primaria basado en el modelo Affective elearning+ josé Antonio Ortega Carrillo, lina María Rendón López, Juan Antonio Fuentes Esparrell y

El objetivo del prototipo de eLearning afectivo fue vertebrar los estados emocionales del alumnado con sus capacidades cognitivas relacionándolos con los objetivos de aprendizaje, para generar respuestas adecuadas para cada alumno atendiendo a estas variables. Este prototipo permite explorar la interacción entre el usuario y el sistema de aprendizaje, para detectar las respuestas emocionales del usuario ante el comportamiento del sistema y, eventualmente, proporcionarle un servicio adaptable y personalizado.

La recopilación de datos afectivos de calidad fue crucial para obtener resultados válidos y fiables: las cámaras y los micrófonos fueron confiables y fáciles de usar, pero hubo que controlar ciertos factores que influyeron en la confiabilidad de los biosensores usados.

Se persiguió que cada sujeto se sintiera durante el experimento sereno y cómodo en el laboratorio, aprendiendo como es habitual en él y procurando que las emociones se generaran de forma natural según el momento del aprendizaje. De esta forma cada sujeto informaba de sus propias emociones cada vez que sentía algún cambio que se registraba en la etiqueta correspondiente.

De esta manera se construyó un prototipo experimental de modelo de aprendizaje electrónico afectivo para mejorar la experiencia de aprendizaje mediante la personalización de la entrega de material didáctico según del estado emocional de cada uno. Se comprobó que existió una superioridad de las emociones conscientes sobre las no conscientes, lo que explicó un aumento de rendimiento del 91\%. No obstante, no se encontraron evidencias para validar el modelo de Kort.

Los autores afirman su deseo de diseñar material de aprendizaje estructurado y recopilar datos de más participantes, para desde ellos perfeccionar su modelo de aprendizaje afectivo en línea implementándolo de forma masiva en la plataforma de aprendizaje de la Universidad de Shanghai, evaluándolo con estudiantes convencionales.

No obstante, hasta la fecha no tenemos constancia de la publicación de nuevos resultados de este equipo precursor del Affective eLearning.

\section{Orígenes y evolución del Modelo Affective eLearning+}

Líderes del actual grupo de Investigación Tecnología Educativa y Educación Social (TEIS) de la Universidad de Granada (España), fueron precursores de la introducción de la educación a distancia mediada por 
Eficacia de un programa de formación en competencias digitales aplicado a estudiantes del grado de magisterio en educación primaria basado en el modelo Affective elearning+ josé Antonio Ortega Carrillo, lina María Rendón lópez, Juan Antonio Fuentes Esparrell y Álvaro Ortega Maldonado

tecnologías digitales en la Universidad de Granada en la década de los años 90 del siglo pasado.

En la segunda década del siglo actual y tras 12 años de realizar evaluaciones de programas de Blended Learning ofertados en la Universidad de Granada y dirigidos por líderes del grupo pusieron de manifiesto que la calidad y satisfacción de los actores intervinientes debía medirse no solo desde los indicadores convencionales sino que debía prestarse una especial atención a la valoración de los flujos emocionales de estos actores como factores decisivos del bienestar y satisfacción general y de su relación con el éxito académico en contextos formativos convencionales y en otros inclusivos y accesibles para personas con discapacidad.

De esta manera fue surgiendo el modelo de eLearning "humanizador, afectivo e inclusivo" que adoptó la denominación de Affective eLearning. En los últimos cinco años, el modelo de ha enriquecido con valiosas aportaciones de la ciencia del bienestar procedentes de la Psicología Positiva, añadiendo el término positivo (+) a la denominación que ha evolucionado a Affective eLearning+. Su arquitectura se asienta en pilares tales como la realización de una cuidadosa evaluación del estado emocional del alumnado antes del comienzo del programa de formación en línea. Ello permite una personalización del e-Learning basada en dinámicas afectivas capaces de generar flujos emocionales que repercutan favorablemente en el bienestar subjetivo de los actores (Hernández-Sánchez y Ortega-Maldonado, 2015). Otros pilares son el diseño y el desarrollo de materiales hipermedia afectivo-positivos, la implementación de un estilo de acompañamiento tutorial emotivo-positivo y la puesta en práctica de un conjunto de estrategias de evaluación afectivo-participativas transparentes que dentro del Campus Virtual Iberoamericano Inclusivo y Accesible. El modelo Affective eLearning+ ha sido implementado y evaluado en programas desarrollados en universidades y estamentos gubernamentales de Argentina, Colombia, España y México (Ortega-Carrillo, \& Ortega Maldonado (2009); Ortega-Carrillo, \& Coicaud (2010); Ramírez-León, \& Ortega-Carrillo (2010); Ortega-Carrillo, \& Peña-Arcila (2011); Ramírez-León y Ortega-Carrillo (2012); Marchisio-Roggiano, \& Ortega-Carrillo (2013); Ortega-Carrillo y Pérez-García (2013); Domínguez-Castillo, Chen , Ortega-Carrillo, \& McCalman (2014); HernándezSánchez y Ortega-Carrillo (2014), López-Burgueño (2014); Ortega-Carrillo, Hernández-Sanchez, Martínez Guzmán, \& Rendón López (2014); Hernández-Sánchez, \& Ortega-Carrillo (2015); Hernández-Sánchez, \& 
Ortega-Carrillo (2016a); Hernández-Sánchez, \& Ortega-Carrillo (2016b); Domínguez-Castillo, Cantó-Herrera, Ortega-Carrillo, \& McCalman (2016); Rojano-Caceres, Morales-Luna, Rebolledo-Mendez, Ortega-Carrillo, Muñoz-Arteaga (2016); Rendón-López, Ortega-Carrillo y OrtegaMaldonado (2017); Ortega-Maldonado, Llorens, Acosta, \& Coo (2017); Pascual-Sevillano, Ortega-Carrillo, Fombona-Cadavieco y Pérez-Ferra (2017); Rendón-Lopez y Ortega-Carrillo (2018); Mireles, Ortega-Carrillo, \& Fuentes-Esparrell (2018).

\section{Marco empírico}

\section{Objetivo}

El objetivo de este trabajo es verificar si el "Programa Interuniversitario de Especialización en Competencias Comunicativo-Digitales", realizado bajo la metodología de "Affective eLearning+" desarrolla competencias digitales en los futuros maestros de Educación Primaria, presentando diferencias significativas respecto al grupo control que no realizó tal programa.

\section{Metodología}

Este trabajo presenta los resultados de la evaluación del conjunto de la población participante en el denominado "Programa Interuniversitario de Especialización en Competencias Comunicativo-Digitales", financiado por el Plan Nacional de I+D+l. Este programa se aplicó a 109 alumnos que cursaban el grado de Educación Primaria en las Universidades de Oviedo, Jaén y en el Centro Universitario SAFA (adscrito a la Universidad de Jaén).

Ofrece el análisis de un cuestionario compuesto por 49 ítems distribuidos en las cuatro dimensiones propias de la competencia digital: Navegación y resolución de problemas técnicos; Disposiciones legales, derechos de autor y licencias; Desarrollo, integración y estructuración de contenidos; Interacción a través de medios digitales y gestión de la identidad digital. Fue aplicado como pretest y postest al conjunto de la población distribuida en los grupos experimental y control. 
Eficacia de un programa de formación en competencias digitales aplicado a estudiantes del grado de magisterio en educación primaria basado en el modelo Affective elearning+ josé Antonio Ortega Carrillo, lina María Rendón lópez, Juan Antonio Fuentes Esparrell y Álvaro Ortega Maldonado

\section{Resultados y discusión}

\section{Fiabilidad del cuestionario usado en el pretest-postest}

Se ha determinado la estabilidad del cuestionario cuando es aplicado a una misma persona en diferentes ocasiones y condiciones similares. El Alfa de Cronbach respecto a la totalidad de la escala es de .786. La eliminación de cada ítem respecto a la totalidad, aporta los siguientes datos (ítem $\mathrm{n}^{\circ} 33=.770<.789=$ ítem $\mathrm{n}^{\circ} 22$ ). De manera que no se suprime ningún ítem, ya que al eliminar respectivamente cada uno de los ítems, el resultado no aporta un alfa superior al de la totalidad de la escala.

Del mismo modo, se aplicó el Alfa de las dos mitades para conocer el nivel de consistencia entre las dos partes del cuestionario. En los ítems impares se obtuvo un alfa de .695, mientras que en los ítems pares un alfa de .710. Por consiguiente, hay una consistencia razonable en la fiabilidad de los ítems pares respecto a los impares.

\section{Contraste de hipótesis entre las opiniones de los estudiantes del grupo control, situación pretest-postest}

Para determinar si se cumplía el "criterio de normalidad" de la curva en los estudiantes que integran el grupo control, en situación pretest-postest, se aplicó la prueba de Kolmogorov-Smirnov, aportando el siguiente índice de significación ( $p=.919>.05$ ); por consiguiente, se cumple el criterio de normalidad de la curva en la distribución de las opiniones de los estudiantes pertenecientes a los grupos control, tanto en situación pretest como postest.

Se aplicó la $t$ de Student para muestras relacionada, ya que se trabaja con el mismo grupo, el grupo control de estudiantes, a fin de comprobar si hubo, o no, alguna variable extraña que incidiese en el proceso, siendo $(t=1.998),(p=.065>.05)$, lo que permite indicar que no hay diferencias estadísticamente significativas entre el grupo control en situación pretest, respecto al grupo control en situación postest, lo que indica que no han intervenido variables extrañas en el proceso.

Contraste de medias (MGC/pretest=3.3016, MGE/postest=3.2593). 
Eficacia de un programa de formación en competencias digitales aplicado a estudiantes del grado de magisterio en educación primaria basado en el modelo Affective elearning+ josé Antonio Ortega Carrillo, lina María Rendón lópez, Juan Antonio Fuentes Esparrell y Álvaro Ortega Maldonado

Tabla 1

$T$ de Student: Diferencia de medias, grupo control, contraste pretest-postest, competencia digital

\begin{tabular}{lccccc}
\hline & Media & $\begin{array}{c}\text { Desviación } \\
\text { estandar }\end{array}$ & t & gl & $\begin{array}{c}\text { Sig } \\
\text { (bilateral }\end{array}$ \\
\hline $\begin{array}{l}\text { Diferencia de medias, grupo con- } \\
\text { trol-pretest/grupo control-postest }\end{array}$ & -.11111 & .76171 & -.357 & 5 & .735 \\
\hline
\end{tabular}

$(p \leq .05)$

Fuente: Elaboración propia

\section{Contraste de hipótesis entre las opiniones de los estudiantes del grupo control-grupo experimental, situación pretest}

Al abordar este aspecto con respecto a la totalidad de la competencia, atendiendo a los grupos control y experimental, en situación pretest, se determinó la prueba de Kolmogorov-Smirnov, a fin de conocer si se cumple el criterio de normalidad en la distribución de la curva. Los resultados aportados indicaron $(p=.999>.05)$, de modo que al no haber diferencias estadísticamente significativas fue posible utilizar pruebas paramétricas.

El estadístico $F$ de la prueba de Levene permite aceptar la igualdad de varianzas poblacionales, $(p=.741>.050)$, determinándose la $t$ de Student en la fila superior. Como puede verse en la tabla 2, el estadístico $t=.004$ y el valor ( $p=.997>.05)$ indicó que no hay diferencias estadísticamente significativas entre el grupo control y el experimental, en situación pretest, competencia digital, razón por la que la aplicación del curso en línea se realizó sin variables que distorsionaran la homogeneidad de medias de partida en ambos grupos (MGC=3.2591, MGE=3.2593).

Tabla 2

$T$ de Student: Contraste grupo control-grupo experimental, situación pretest, competencia digital

\begin{tabular}{|c|c|c|c|c|c|c|c|}
\hline & \multicolumn{4}{|c|}{$\begin{array}{c}\text { Prueba de } \\
\text { Levene }\end{array}$} & \multicolumn{3}{|c|}{ Prueba $t$ para la igualdad de medias } \\
\hline & $\mathrm{F}$ & Sig. & $\mathrm{t}$ & $\mathrm{gl}$ & $\begin{array}{c}\text { Sig. } \\
\text { bilateral }\end{array}$ & $\begin{array}{l}\text { Dif. de Dif. error } \\
\text { medias estándar }\end{array}$ & $\frac{95 \% \text { de int. conf }}{\text { Inferior Superior }}$ \\
\hline $\begin{array}{l}\text { Se asumen va- } \\
\text { rianzas iguales } \\
\end{array}$ & .110 & .741 & .004 & 557 & .997 & .05105 & -.10010 .10047 \\
\hline $\begin{array}{l}\text { No se asumen } \\
\text { var. iguales }\end{array}$ & & & .004 & 425.782 & .997 & .05095 & -.09996 .10033 \\
\hline
\end{tabular}

Fuente: Elaboración propia 
Eficacia de un programa de formación en competencias digitales aplicado a estudiantes del grado de magisterio en educación primaria basado en el modelo Affective elearning+ josé Antonio Ortega Carrillo, lina María Rendón López, Juan Antonio Fuentes Esparrell y Álvaro Ortega Maldonado

\section{Contraste de hipótesis entre las opiniones de los estudiantes del grupo grupo-control, grupo-experimental, en situación postest, respecto a la competencia digital}

Se pretende conocer si la aplicación del programa en el que han participado los estudiantes de primer curso de Magisterio del Título de Educación Primaria ha generado un mayor dominio de la competencia digital (en sus correspondientes unidades de competencia y respectivos criterios de desempeño) en los estudiantes del grupo experimental, que han seguido el programa de mejora, respecto a los del grupo control que no lo siguieron.

Al abordar este aspecto, respecto a la totalidad de la competencia digital, comparando el grupo control con el experimental, se realizó la prueba de Kolmogorov-Smirnov, a fin de conocer si se cumple el criterio de normalidad en la distribución de las opiniones de los estudiantes en la curva. Los resultados aportados $(p=.234=. \geq .05)$ indicaron que no hay diferencias estadísticamente significativas y, por tanto, se cumple el mencionado criterio, razón por la que se utilizarán pruebas paramétricas. Al ser las variables dicotómicas, se empleó la $t$ de Student, en este caso, para muestras independientes, ya que los grupos son diferentes.

Se determinó si la aplicación del programa elaborado a tal fin tuvo consecuencias en el grupo experimental, en situación postest, respecto al grupo control, en relación con la competencia digital.

El estadístico $F$ de la prueba de Levene aporta diferencias significativas $y$, por tanto, no se cumple la homogeneidad de varianzas de entre los dos grupos comparados $(p=.001<.05)$; por consiguiente, se trabajó con los datos de la fila inferior. Como puede apreciarse en la tabla 3, el estadístico $t=10.673$ y el valor $(p=.000<.05)$, ratifican que hay diferencias estadísticamente significativas entre en el grupo control y el grupo experimenta en situación postest, medias (MGC=2.7484, MGE=3.2647).

Para mostrar la consistencia de estas diferencias y la inferencia que tiene el curso aplicado sobre la mejora de la competencia digital, se acudió a la potencia estadística y al tamaño del efecto. Respecto a la potencia estadística $(1-\beta)=1.000$, el resultado indica que se rechaza la hipótesis nula, así como que esta situación se cumple en el 100 \% de los casos.

Respecto al tamaño del efecto $(d)$, se pone de manifiesto que $(d)=.453$, lo que evidencia que estamos ante una inferencia media-baja de la variable independiente sobre la dependiente. 
Eficacia de un programa de formación en competencias digitales aplicado a estudiantes del grado de magisterio en educación primaria basado en el modelo Affective elearning+ josé Antonio Ortega Carrillo, lina María Rendón López, Juan Antonio Fuentes Esparrell y Álvaro Ortega Maldonado

Tabla 3

Contraste de hipótesis entre las opiniones de los estudiantes de los grupos control-grupo experimental, en situación postest, respecto a la totalidad de dimensiones que integran la competencia digital

\begin{tabular}{|c|c|c|c|c|c|c|c|c|}
\hline \multirow{2}{*}{$\mathrm{F}$} & \multicolumn{3}{|c|}{$\begin{array}{c}\text { Prueba de } \\
\text { Levene }\end{array}$} & \multicolumn{5}{|c|}{ Prueba t para la igualdad de medias } \\
\hline & Sig. & $\mathrm{t}$ & gl & $\begin{array}{c}\text { Sig. } \\
\text { bilateral }\end{array}$ & $\begin{array}{l}\text { Dif. de } \\
\text { medias }\end{array}$ & $\begin{array}{l}\text { Dif. error } \\
\text { estándar }\end{array}$ & $\frac{95 \% \text { de }}{\text { Inferior }}$ & int. conf \\
\hline $\begin{array}{l}\text { Se asumen } \\
\text { varianzas } \\
\text { iguales }\end{array}$ & $20.632,000$ & 11.122 & 237 & .000 & .84143 & .07565 & .69239 & .99046 \\
\hline $\begin{array}{l}\text { No se asu- } \\
\text { men var. } \\
\text { iguales }\end{array}$ & & 10.656 & 68.036 & .000 & .84143 & .07896 & .68554 & .99732 \\
\hline
\end{tabular}

Fuente: Elaboración propia

Se pretende conocer si la aplicación del programa en el que han participado los estudiantes ha generado un mayor dominio de la competencia digital en sus 4 dimensiones y criterios de desempeño de cada una de ellas, en los estudiantes del grupo experimental, respecto a los del grupo control.

\section{Contraste de hipótesis entre las opiniones de los estudiantes del grupo control-grupo experimental, en situación postest, respecto a la unidad competencia navegación y resolución de problemas técnicos.}

Se determinó la prueba de Kolmogorov-Smirnov, a fin de conocer si se cumple el criterio de normalidad en la distribución de las opiniones de los estudiantes en la curva. Los resultados aportados $(p=.108 \geq .05)$ indicaron que no hay diferencias estadísticamente significativas y, por tanto, se cumple el mencionado criterio, razón por la que se utilizarán pruebas paramétricas. Al ser las variables dicotómicas, se empleará la $t$ de Student para muestras independientes, ya que los grupos son diferentes.

Se determinó si la aplicación del programa tuvo consecuencias en el grupo experimental, en situación postest, respecto al grupo control, como consecuencia de la aplicación del programa referenciado, en relación con la competencia digital.

El estadístico $F$ de la prueba de Levene aporta diferencias significativas $y$, por tanto, no se cumple la homogeneidad de varianzas de entre 
Eficacia de un programa de formación en competencias digitales aplicado a estudiantes del grado de magisterio en educación primaria basado en el modelo Affective elearning+ josé Antonio Ortega Carrillo, lina María Rendón lópez, Juan Antonio Fuentes Esparrell y Álvaro Ortega Maldonado

los dos grupos comparados $(p=.000<.05)$; por consiguiente, se trabajó con los datos de la fila inferior. Como puede apreciarse en la tabla 4, el estadístico $(t=8.788)$ y el valor $(p=.000<.05)$, ratifica que hay diferencias estadísticamente significativas entre en el grupo control y el grupo experimental $(\mathrm{MGC}=2.8758, \mathrm{MGE}=3.6920)$.

Tabla 4

Contraste de hipótesis entre las opiniones de los estudiantes del grupo controlgrupo experimental, en situación postest, respecto a la unidad de competencia, navegación y resolución de problemas técnicos

\begin{tabular}{|c|c|c|c|c|c|c|c|}
\hline \multirow{2}{*}{ 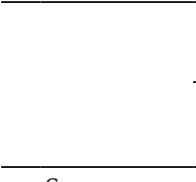 } & \multicolumn{3}{|l|}{$\begin{array}{c}\text { Prueba de } \\
\text { Levene }\end{array}$} & \multicolumn{4}{|c|}{ Prueba t para la igualdad de medias } \\
\hline & F Sig. & $\mathrm{t}$ & gl & $\begin{array}{l}\text { Sig. bi- } \\
\text { lateral }\end{array}$ & $\begin{array}{l}\text { Dif. de } \\
\text { medias }\end{array}$ & $\begin{array}{l}\text { Dif. error } \\
\text { estándar }\end{array}$ & $\frac{95 \% \text { de int. conf }}{\text { Inferior Superior }}$ \\
\hline $\begin{array}{l}\text { Se asumen } \\
\text { varianzas } \\
\text { iguales }\end{array}$ & $24.896,000$ & 6.647 & 488 & .000 & .48 .410 & .07283 & 62720. \\
\hline $\begin{array}{l}\text { No se asu- } \\
\text { men var. } \\
\text { iguales }\end{array}$ & & 7.903 & 34.364 & .000 & .48410 & .06126 & $\begin{array}{ll}.36360459 & .6045\end{array}$ \\
\hline
\end{tabular}

Fuente: Elaboración propia

Para mostrar la consistencia de estas diferencias y la inferencia que tiene el curso aplicado sobre la mejora de la competencia digital, se acudió a la potencia estadística y al tamaño del efecto. Respecto al tamaño del efecto, los resultados fueron $(1-\beta)=1.000$, que indica que se rechaza la hipótesis nula siempre, así como que esta situación se cumple en el $100 \%$ de los casos. Respecto al tamaño del efecto $(d)$, se pone de manifiesto que $(d)=.346$, lo que evidencia que estamos ante una posibilidad baja de inferencia de la variable independiente, materializada en el curso aplicado, sobre la variable dependiente, competencia digital.

En relación con los criterios de desempeño que se integran en torno a la unidad de competencia "navegación y resolución de problemas técni$\cos ^{\prime \prime}$, se evidencian diferencias estadísticamente significativas respecto a los ítems: 1, 10, 37, 38 y 47, que comparte el resultado $(p=.000<.050)$. Sigue habiendo diferencias estadísticamente significativas en los ítems $44(p=.008>.050)$ y $45(p=.015>.050)$, pero menos acusadas; por consiguiente, el curso en línea que se ha aplicado ha generado mejoras en los estudiantes del grupo experimental, respecto a su capacidad para configurar el navegador web de sus ordenadores y han mejorado en la 
capacidad de utilizar espacios de almacenamiento externo. Del mismo modo, en la capacidad de realizar operaciones básicas de protección y mantenimiento de los dispositivos que utilizan: cómo actuar cuando detectan una amenaza de seguridad en los dispositivos o cómo acceder a foros especializados que les faciliten información. Sin embargo, no han experimentado mejora en la detección, explicación de problemas técnicos, ni en su resolución, aspectos que son de mayor complejidad y demandas una formación más específica.

\section{Contraste de hipótesis entre las opiniones de los estudiantes del grupo control-grupo experimental, en situación postest, respecto a la unidad competencia disposiciones legales, derechos de autor y licencias}

Se intentó conocer si la aplicación del programa para la mejora de la unidad de competencia, "disposiciones legales, derechos de autor y licencias" mejora en los estudiantes del grupo experimenta, en relación con los del grupo control, como consecuencia de la aplicación del curso realizado a tal fin.

Se determinó la prueba de Kolmogorov-Smirnov, a fin de conocer si se cumple el criterio de normalidad en la distribución de las opiniones de los estudiantes en la curva. Los resultados aportados $(p=.078 \geq .05)$, indicaron que no hay diferencias estadísticamente significativas $y$, por tanto, se cumple el mencionado criterio, razón por la que se utilizarán pruebas paramétricas. Empleándose la $t$ de Student para muestras independientes.

El estadístico $F$ de la prueba de Levene aporta diferencias estadísticamente significativas y, por tanto, no se cumple la prueba de homocedasticidad, razón por la que no hay homogeneidad de varianzas entre los grupos contrastados, $(p=.000<.05)$; por consiguiente, se trabajó con los datos de la fila inferior. Como puede apreciarse en la tabla 5, el estadístico $(t=8.396)$ y el valor $(p=.000<.05)$ ratifican que hay diferencias estadísticamente significativas entre ambos grupos, $(M G C=2.5835$, MGE=3.4391). 
Eficacia de un programa de formación en competencias digitales aplicado a estudiantes del grado de magisterio en educación primaria basado en el modelo Affective elearning+ josé Antonio Ortega Carrillo, lina María Rendón lópez, Juan Antonio Fuentes Esparrell y Álvaro Ortega Maldonado

Tabla 5

Contraste de hipótesis entre las opiniones de los estudiantes del grupo controlgrupo experimenta, en situación postest, respecto a la unidad de competencia disposiciones legales, derechos de autor y licencias

\begin{tabular}{|c|c|c|c|c|c|c|c|}
\hline & \multicolumn{4}{|c|}{$\begin{array}{l}\text { Prueba de } \\
\text { Levene }\end{array}$} & \multicolumn{3}{|c|}{ Prueba t para la igualdad de medias } \\
\hline & $\mathrm{F}$ & Sig. & $\mathrm{t}$ & gl & $\begin{array}{l}\text { Sig. bi- Dif. de } \\
\text { lateral medias }\end{array}$ & $\begin{array}{l}\text { Dif. error } \\
\text { estándar }\end{array}$ & $\frac{95 \% \text { de int. conf }}{\text { Inferior Superior }}$ \\
\hline $\begin{array}{l}\text { Se asumen } \\
\text { varianzas } \\
\text { iguales }\end{array}$ & 30.382 &, 000 & 8.748 & 237 & .38831 & .85554 & .097801 .04821 \\
\hline $\begin{array}{l}\text { No se asu- } \\
\text { men var. } \\
\text { iguales } \\
\end{array}$ & & & 8.3961 & 70.772 & .38831 & .85554 & .101901 .05669 \\
\hline
\end{tabular}

Fuente: Elaboración propia

Para mostrar la consistencia de estas diferencias y la inferencia que tiene el curso aplicado sobre la mejora de la competencia digital, se acudió a la potencia estadística y al tamaño del efecto. Respecto al tamaño del efecto, los resultados fueron $(1-\beta)=.992$, que indica que se rechaza la hipótesis nula, así como que esta situación se cumple en el 99\% de los casos; es decir, casi siempre. Sin embargo, respecto al tamaño del efecto $(d)$, se pone de manifiesto que $(d)=.686$, evidenciando una inferencia media del curso aplicado sobre la mejora de la unidad de competencia "disposiciones legales, derechos de autor y licencias".

En relación a los criterios de desempeño que integran la mencionada unidad de competencia hay diferencias estadísticamente significativas con un estadígrafo de $(p=.000<.050)$, respecto a los criterios de desempeño: 14, 22, 23, 33, 34 y 35; por consiguiente, el programa aplicado ha logrado mejoras sustantivas, respecto a: "compartir información" o "contenidos obtenidos en línea"; "cumplimiento de las normas legales que regulan la transferencia de información"; "consideración de las características socioculturales de la audiencia hacia la que se dirigen nuestros mensajes"; "mejora en el cumplimiento de las normas legales que regulan la transferencia de información". También se ha mejorado sobre "el respeto y cumplimiento de las obligaciones y derechos previstos en la normativa que regula las licencias de uso de materiales creados por otros autores", así como "la preocupación por conocer los procedimientos por los cuales se pueden liberalizar los derechos de autor". También 
hay diferencias ente el grupo control y experimental, a favor del segundo, generadas por el programa de intervención aplicado; sin embargo, son menos acentuadas que en los criterios de desempeño anteriores. Diferencias que se concretan en "el cumplimiento de las normas legales que regulan la transferencia de información, cuando se emiten o reciben mensajes" $(p=.003<.05)$.

\section{Contraste de hipótesis entre las opiniones de los estudiantes del grupo control-grupo experimental, en situación postest, respecto a la unidad competencia desarrollo, integración y estructuración de contenidos}

Otra de las unidades de competencia sobre las que se quiso determinar si hubo diferencias estadísticamente significativas entre el grupo control y experimental, en situación postest, quedó definida en la unidad de competencia "desarrollo, integración y estructuración de contenidos".

Se determinó la prueba de Kolmogorov-Smirnov, a fin de conocer si se cumple el criterio de normalidad en la distribución de las opiniones de los estudiantes en la curva. Los resultados aportados ( $p=.066 \geq .05)$, indica que no hay diferencias estadísticamente significativas $y$, por tanto, se cumple el mencionado criterio, razón por la que se utilizarán pruebas paramétricas. Al ser las variables dicotómicas se empleó en este caso la $t$ de Student para muestras independientes, ya que las muestras son diferentes.

El estadístico $F$ de la prueba de Levene aporta diferencias estadísticamente significativas $y$, por tanto, no se cumple la homogeneidad de varianzas entre los dos grupos comparados $(p=.000<.05)$; por consiguiente, se trabajó con los datos de la fila inferior. Como puede apreciarse en la tabla 6 , el estadístico $(t=1.646)$ y el valor $(p=.102>.05)$ ratifican que no hay diferencias estadísticamente significativas entre el grupo control y el experimental (MGC=2.7096, MGE=2.9358); es decir, el programa no ha reportado efecto de mejora respecto a la mencionada unidad de competencia. 
Eficacia de un programa de formación en competencias digitales aplicado a estudiantes del grado de magisterio en educación primaria basado en el modelo Affective elearning+ josé Antonio Ortega Carrillo, lina María Rendón lópez, Juan Antonio Fuentes Esparrell y Álvaro Ortega Maldonado

Tabla 6

Contraste de hipótesis entre las opiniones de los estudiantes del grupo controlgrupo experimenta, en situación postest, respecto a la unidad de competencia interacción a través de medios digitales y gestión de la identidad digital

\begin{tabular}{|c|c|c|c|c|c|c|c|}
\hline & \multicolumn{3}{|l|}{$\begin{array}{c}\text { Prueba de } \\
\text { Levene }\end{array}$} & \multicolumn{4}{|c|}{ Prueba t para la igualdad de medias } \\
\hline & Sig. & $\mathrm{t}$ & gl & $\begin{array}{l}\text { Sig. bi- Dif. de } \\
\text { lateral medias }\end{array}$ & $\begin{array}{l}\text { Dif. error } \\
\text { estándar }\end{array}$ & $\frac{95 \% \text { de }}{\text { Inferior }}$ & $\begin{array}{l}\text { int. conf } \\
\text { Superior }\end{array}$ \\
\hline $\begin{array}{l}\text { Se asumen } \\
\text { varianzas } \\
\text { iguales }\end{array}$ & 24.614 .000 & 1.701 & 273 & .22616 & .22616 & -.03577 & .48810 \\
\hline $\begin{array}{l}\text { No se asu- } \\
\text { men var. } \\
\text { iguales }\end{array}$ & & 1.646 & 183.611 & .22616 & .22616 & .04500 & .49733 \\
\hline
\end{tabular}

Fuente: Elaboración propia

Los resultados aportados en el contraste de medias no permiten realizar el la potencia estadística ni el tamaño del efecto.

Los resultados aportados por el contraste de hipótesis en los cuatro criterios de desempeño que aporta esta unidad de competencia son desiguales, ya que mientras que el programa aplicado no ha generado diferencias significativas entre el grupo control y el grupo experimental en el avance relativo a mejorar la gestión de espacios web donde publicar contenidos multimedia ( $p=.910>.05)$ o en la creación de audios digitales mezclando voz y música ( $p=.649>.05)$, sí se han producido en otros dos criterios de desempeño, ya que los estudiantes del grupo experimental han mejorado en la creación de videos propios insertando fotografías, gráficos, etc., $(p=.020<.05)$ y en el uso de programas de edición digital de audio y/o video para modificar contenidos $(p=.005<.050)$

\section{Contraste de hipótesis entre las opiniones de los estudiantes del grupo control-grupo experimental, en situación postest, respecto a la unidad de competencia, interacción a través de medios digitales y gestión de la identidad digital}

Se intenta conocer si la aplicación del curso para la mejora de la unidad de competencia, "interacción a través de medios digitales y gestión de la identidad digital" mejora el dominio de dicha unidad de competencia en los estudiantes del grupo experimental, en relación con los del grupo control. 
Eficacia de un programa de formación en competencias digitales aplicado a estudiantes del grado de magisterio en educación primaria basado en el modelo Affective elearning+ josé Antonio Ortega Carrillo, lina María Rendón López, Juan Antonio Fuentes Esparrell y

Al abordar este aspecto se determinó la prueba de Kilmogorov-Smirnov, a fin de conocer si se cumple el criterio de normalidad en la distribución de las opiniones de los estudiantes en la curva. Los resultados aportados $(p=.058 \geq .05)$, indicaron que no hay diferencias significativas $y$, por tanto, se cumple el mencionado criterio, razón por la que se utilizaron pruebas paramétricas. Al ser las variables dicotómicas, se empleó la $t$ de Student, para muestras independientes, ya que los grupos contrastados son diferentes.

El estadístico $F$ de la prueba de Levene no aporta diferencias estadísticamente significativas $y$, por tanto, se cumple la homogeneidad de varianzas entre los dos grupos comparados $(p=.099<.05)$; por consiguiente se trabajó con los datos de la fila superior. Como puede apreciarse en la tabla 7 , el estadístico $(t=15.523)$ y el valor $(p=.000<.05)$ ratifican que hay diferencias estadísticamente significativas entre ambos grupos, (MGC=2.8872, MGE=4.5749), comprobándose acusadas diferencias entre los estudiantes del grupo control respecto al experimental.

\section{Tabla 7}

Contraste de hipótesis entre las opiniones de los estudiantes del grupo controlgrupo experimental, en situación postest, respecto a la unidad de competencia, interacción a través de medios digitales y gestión de la identidad digital

$$
\begin{aligned}
& \begin{array}{l}
\text { Prueba de } \\
\text { Levene }
\end{array} \text { Prueba t para la igualdad de medias } \\
& \hline
\end{aligned}
$$

\begin{tabular}{|c|c|c|c|c|c|c|}
\hline & & Sig. & & $\begin{array}{l}\text { Sig. bi- } \\
\text { lateral }\end{array}$ & $\begin{array}{l}\text { Dif. de Dif. erro } \\
\text { medias estándar }\end{array}$ & $\frac{95 \% \text { de int. conf }}{\text { Inferior Superior }}$ \\
\hline & $\begin{array}{l}\text { Se asumen } \\
\text { varianzas } \\
\text { iguales }\end{array}$ & $30.382,000$ & 4.371 & .000 & 08884. 38831. & .21375.56286 \\
\hline $\mathrm{rl}$ & $\begin{array}{l}\text { No se asu- } \\
\text { men var. } \\
\text { iguales }\end{array}$ & & \multicolumn{2}{|c|}{5.512386 .376 .000} & . 07044. & .24981.52681 \\
\hline
\end{tabular}

Fuente: Elaboración propia

Para mostrar la consistencia de estas diferencias y la inferencia que tiene el curso aplicado, variable dependiente, sobre la mejora de la competencia digital, se acudió a la potencia estadística y al tamaño del efecto, respectivamente. Respecto al tamaño del efecto, los resultados fueron $(1-\beta)=1.000$, que indica que se rechaza la hipótesis nula, así como que esta situación se cumple en el $100 \%$ de los casos; es decir, siempre. Sin embargo, respecto al tamaño del efecto se pone de manifiesto que $(d)=.718$, lo que evidencia inferencia media-media del curso aplicado 
Eficacia de un programa de formación en competencias digitales aplicado a estudiantes del grado de magisterio en educación primaria basado en el modelo Affective elearning+ josé Antonio Ortega Carrillo, lina María Rendón lópez, Juan Antonio Fuentes Esparrell y Álvaro Ortega Maldonado

sobre la mejora de la unidad de competencia "interacción a través de medios digitales y gestión de la identidad digital".

Los criterios de desempeño que se integran en torno a la unidad de competencia "interacción a través de medios digitales y gestión de la identidad digital", evidencia diferencias significativas entre el grupo control y el experimental. Así, el criterio de desempeño "utiliza, al menos, tres herramientas digitales distintas para interactuar con los demás, ha experimentado un avance en los estudiantes del grupo experimental $(p=.000<.05)$; la interacción con diferentes fuentes de información ha aumentado cuando navegan los estudiantes $(p=.000<.05)$, lo que también sucede es el aumento de la capacidad de los estudiantes del grupo experimental de gestionar su identidad, cuidando de hacer público solo los datos personales que desean que se conozcan; por consiguiente, se comprueban avances en el dominio de la unidad de competencia de referencia.

La tabla adjunta muestra el balance claramente favorable de las diferencias significativas obtenidas en la mayor parte de las unidades de desempeño analizadas (16), es decir el $80 \%$, frente aquellas otras que no las han obtenido (4) equivalentes a un $20 \%$, lo que subraya la alta eficacia del programa de formación en línea implementado con la ayuda de los materiales didácticos y la metodología propios del modelo Affective eLearning+, creado anteriormente a este programa por el Grupo de Investigación "Tecnología Educativa e Investigación Social" (TEIS) de la Universidad de Granada.

Tabla 8

Ámbitos de competencia digital y unidades de desempeño

\section{Navegación y resolución de problemas técnicos}

Desempeños con diferencias sig. prets- $\quad$ Desempeños sin diferencias sig. postst. prets-postst.

Capacidad para configurar el navegador Detección, explicación de proweb. blemas técnicos y su resolución.

Capacidad de utilizar espacios de almacenamiento externo.

Capacidad de realizar operaciones básicas de protección y mantenimiento de los dispositivos usados

Adecuada actuación cuando detectan una amenaza de seguridad en los dispositivos.

Acceso a foros especializados de información. 
Eficacia de un programa de formación en competencias digitales aplicado a estudiantes del grado de magisterio en educación primaria basado en el modelo Affective elearning+ josé Antonio Ortega Carrillo, lina María Rendón López, Juan Antonio Fuentes Esparrell y Álvaro Ortega Maldonado

\section{Disposiciones legales, derechos de autor y licencias}

Compartir información o contenidos obte- Cumplimiento de las normas lenidos en línea. gales que regulan la transferencia de información, cuando se emiten o reciben mensajes.

Preocupación por conocer los procedimientos por los cuales se pueden liberalizar los derechos de autor.

Cumplimiento de las normas legales que regulan la transferencia de información.

Consideración de las características socioculturales de la audiencia hacia la que se dirigen nuestros mensajes.

Mejora en el cumplimiento de las normas legales que regulan la transferencia de información.

Respeto y cumplimiento de las obligaciones y derechos previstos en la normativa que regula las licencias de uso de materiales creados por otros autores.

Desarrollo, integración y estructuración de contenidos

Creación de videos propios insertando foto- Mejorar la gestión de espacios grafías, gráficos, etc. web para publicar contenidos multimedia.

Uso de programas de edición digital de au- Creación de audios digitales dio y/o video para modificar contenidos. mezclando voz y música.

\section{Interacción a través de medios digitales y gestión de la identidad digital}

Utilizan al menos, tres herramientas digitales distintas para interactuar con los demás. Interacción con diferentes fuentes de información.

Capacidad de gestionar su identidad, cuidando de hacer público solo los datos personales que desean que se conozcan de referencia.

Fuente: elaboración propia.

\section{Discusión de resultados}

En lo referido al pretest, y atendiendo a los grupos control y experimental, en situación pretest, se determinó mediante la la prueba de Kolmogorov- 
Eficacia de un programa de formación en competencias digitales aplicado a estudiantes del grado de magisterio en educación primaria basado en el modelo Affective elearning+ josé Antonio Ortega Carrillo, lina María Rendón lópez, Juan Antonio Fuentes Esparrell y Álvaro Ortega Maldonado

Smirnov, la no existencia de diferencias estadísticamente significativas lo que permitió el uso de pruebas paramétricas.

El estadístico $\mathrm{F}$ de la prueba de Levene indicó la no existencia de diferencias estadísticamente significativas entre el grupo control y el experimental, en situación pretest de la competencia digital, por lo que la que la aplicación del curso en línea se realizó sin variables que distorsionaran la homogeneidad de medias de partida en ambos grupos.

Para determinar si la aplicación del programa de entrenamiento educativo mejoró la formación en competencia digital del grupo experimental se pasó el postest respecto al grupo control y se aplicó la prueba de Kolmogorov-Smirnov, a fin de conocer si se cumplía el criterio de normalidad en la distribución de las opiniones de los estudiantes en la curva. Los resultados aportados indicaron la no existencia de diferencias estadísticamente significativas por lo que se cumplió tal criterio y con ello se pudieron utilizará pruebas paramétricas.

El estadístico F de Levene aportó diferencias significativas no existiendo homogeneidad de varianzas entre los dos grupos. Los resultados obtenidos ratifican las diferencias estadísticamente significativas entre en el grupo control y el grupo experimental. Su potencia estadística permite rechazar la hipótesis nula si bien estos resultados ponen de manifiesto que existe inferencia variable un tanto dispar, en unos caos sustancial o mediamedia, en otros media-baja y en contados casos nula, de la variable independiente sobre la dependiente en los ítems que miden las competencias que componen las cuatro unidades analizadas: navegación y resolución de problemas técnicos; disposiciones legales, derechos de autor y licencias; desarrollo, integración y estructuración de contenidos y, finalmente, interacción a través de medios digitales y gestión de la identidad digital.

\section{Conclusiones}

Las diferencias significativas obtenidas al contrastar los resultados del alumnado del grado de Magisterio de Educación Primaria en el programa en línea de alfabetización en competencia digital basado en el modelo Affective eLearning+ y del alumnado que actuó como grupo de control presentan un balance claramente favorable en 9 de los 13 desempeños competenciales trabajados es decir del 70\% de las competencias evaluadas. 
Eficacia de un programa de formación en competencias digitales aplicado a estudiantes del grado de magisterio en educación primaria basado en el modelo Affective elearning+ josé Antonio Ortega Carrillo, lina María Rendón López, Juan Antonio Fuentes Esparrell y Álvaro Ortega Maldonado

Tales resultados ponen de manifiesto que el modelo Affective eLearnig+ anteriormente aplicado y validado en otros programas de formación del profesorado tanto en España, como en otros países tales como Argentina, Colombia y México es eficaz para desarrollar competencias profesionales en un clima de bienestar y satisfacción. Los materiales didácticos hipermedia emotivo-positivos creados para este programa de alfabetización digital junto al desarrollo de la tutoría afectivo-positiva (señas de identidad clave del modelo), así como el adecuado uso de las herramientas de comunicación digital positiva usadas en el Campus Virtual Iberoamericano Inclusivo y Accesible en el que se aplica el modelo, han sido componentes innovadores propios de la web 4.0 que han probado una vez más su eficiencia en programas de formación línea de naturaleza "humanizadora", afectivo-positiva e inclusiva.

\section{Referencias}

Domínguez-Castillo, Chen, A., Ortega-Carrillo, J. A. \& McCalman, D. (2014). Digital communication technologies for teachers of science and mathematics in México. Jurnal of Instrutional Pedagogies. (14). 1941-3394.

Domínguez-Castillo, J. G., Cantó-Herrera, P. J., Ortega-Carrillo, J. A. \& McCalman, D.G. (2016). Raising the technological competence of high school science and mathematics teachers of México through delivery of an online program. International Journal Technology, Policy and Managemen,16(2),163-180. DOI: https://doi.org/10.1504/ IJTPM.2016.076317.

Hernández-Sánchez, A .M. \& Ortega-Carrillo, J.A., (2016a). Percepción de bienestar en experiencias inclusivas de blended learning. Educatio Siglo XXI, Vol. 34(2) 63-82. DOI: https://doi.org/10.6018/j/263811

Hernández-Sánchez, A. M. \& Ortega-Carrillo, J. A. (2016b). Aprendiendo en línea lengua de signos española: análisis de la satisfacción del alumnado sordo y oyente en una experiencia realizada en la modalidad semipresencial. Profesorado, 20(2), 350365. https://recyt.fecyt.es/index.php/profesorado/article/view/52110

Hernández-Sánchez, A. M. y Ortega-Carrillo, J.A. (2014). El análisis de contenido de descripciones autobiográficas como estrategia de mejora de un programa de aprendizaje de la lengua de signos española en un campus virtual inclusivo y accesible. Quaderns digital.net. http://www.quadernsdigitals.net/index.php?accionMenu=hemeroteca. VisualizaArticulolU.visualiza\&articulo_id $=11336 \&$ PHPSESSID $=57 \mathrm{ccab81dcf12aa6d}$ Od45e99e771885c

Hernández-Sánchez, A. M. y Ortega-Maldonado, A. (2015). Hacia la personalización del e-Learning: la afectividad y su repercusión en el bienestar subjetivo. Revista LasaIlista de Investigación, 12(2), 194-203. DOI: http://dx.doi.org/10.22507/rli.v12n2a20 Hernández-Sánchez, A.M. \& Ortega-Carrillo, J. A. (2015). Aprendizaje electrónico afec- 
Eficacia de un programa de formación en competencias digitales aplicado a estudiantes del grado de magisterio en educación primaria basado en el modelo Affective elearning+ josé Antonio Ortega Carrillo, lina María Rendón lópez, Juan Antonio Fuentes Esparrell y Álvaro Ortega Maldonado

tivo: un modelo innovador para desarrollar una acción tutorial virtual de naturaleza inclusiva. Formación Universitaria, 2 (VIII), 19-26. DOI: http://dx.doi.org/10.4067/ S0718-50062015000200004

Kort, B.; Reilly, R. \& Picard, R. W. (2001). An affective model of interplay between emotions and learning: Reengineering educational pedagogy-building a learning companion. Proceedings of the IEEE International Conference on Advanced Learning Technologies. Los Alamitos: CA: IEEE ComputerSociety Press, 43-46

López-Burgueño, T. (2014). La música y el bienestar emocional en la educación a distancia desde el modelo Affective e-Learning+. Revista Etic@net, 14(2) 198-226. Recuperado de http://eticanet.org/revista/index.php/eticanet/article/view/54/49

Marchisio-Roggiano, S. \& Ortega-Carrillo, J. A. (2013). Educación virtual afectiva en la formación de investigadores. Etic@net, 13(2), 379-398. http://eticanet.org/revista/ index.php/eticanet/article/view/40

Mireles, M.C., Ortega-Carrillo, J. A, \& Fuentes-Esparrell, J.A. (2018). Equipamiento, formación y usos del teléfono móvil en estudiantes de doctorado de la Universidad Pedagógica Experimental Libertador. PixelBits, (52), 229-243. DOI: https://doi. org/10.12795/pixelbit.2018.i52.16

Ortega-Carrillo, J. A. \& Coicaud, S. M., (2010). Construyendo motivaciones diferentes para estudiar en la universidad. la formación de enfermeros a distancia en la universidad nacional de la Patagonia. Pixel-Bit, (36), 181-192. Recuperado de: https://dialnet. unirioja.es/servlet/articulo?codigo $=3178050$

Ortega-Carrillo, J. A. \& Ortega Maldonado, A. (2009). La comunicación didáctica digital en el espacio europeo de educación superior. Icono 14, 14, 64-83. Recuperado de: https://icono14.net/ojs/index.php/icono14/article/view/303

Ortega-Carrillo, J. A. \& Peña-Arcila, J. B., (2011). Los metaversos como micro ciudades digitales: experiencias de movilidad virtual en el espacio académico américa latina-caribe unión europea realizadas desde la red euro iberoamericana ECAEVAVIRTUALCUE. Actas icono14 - II congreso internacional ciudades creativas, revista de comunicación y nuevas tecnologías, pp. 1457-1470. Recuperado de http://www. scribd.com/doc/111056543/Actas-del-II-Congreso-Ciudades-Creativas-Tomo-3.

Ortega-Carrillo, J. A. y Pérez-García, A. (2013). El cine digital en la formación inicial del profesorado: una experiencia innovadora realizada en la universidad de Granada. Educación XX1, 16(2), 297-319.

Ortega-Carrillo, J. A., Hernández-Sanchez, A., Martínez Guzmán, M., \& López Rendón, L. (2014). La educación a distancia en entornos virtuales hoy. Granada-Bogotá y Guadalajara: Grupo Editorial Universitario (Edición simultánea en España, Colombia y México).

Ortega-Maldonado, A., Llorens, S., Acosta, H., \& Coo, C. (2017). Face-to-Face vs Online: An analysis of Profile, Learning, Performance and Satisfaction among Post Graduate Students. Universal Journal of Educational Research, 5(10), 1701 - 1706 DOI: 10.13189/ujer.2017.051005. Recuperado de https://files.eric.ed.gov/fulltext/ EJ1155533.pdf

Pascual-Sevillano, M. A. Ortega-Carrillo, J. A., Fombona-Cadavieco, J. y Pérez-Ferra, M. (2017). Diseño y validación de un cuestionario sobre la percepción de la competen- 
Eficacia de un programa de formación en competencias digitales aplicado a estudiantes del grado de magisterio en educación primaria basado en el modelo Affective elearning+ josé Antonio Ortega Carrillo, lina María Rendón López, Juan Antonio Fuentes Esparrell y Álvaro Ortega Maldonado

cia digital en estudiantes de $1^{\circ}$ curso del grado de educación primaria. En J. RuizPalmero, J. Sánchez-Rodríguez, S y E. Sánchez Rivas (Coords). Innovación docente y uso de las TIC en educación: Las TIC en la formación, desarrollo de competencias, otras metodologías. Málaga: UMA Editorial.

Picard, R. W. (1997). Affective computing, Boston: MIT Press.

Ramírez-León, Y. \& Ortega-Carrillo, J. A. (2010). Diseños Didácticos Adaptativos a los Estilos de Aprendizaje en Plataformas de Teleformación. En García, J. y otros (Eds.) Actas del IV Congreso Mundial de estilos de aprendizaje (pp. 447- 462). México: Colegio de Postgraduados Montecillo.

Ramírez-León, Y. y Ortega-Carrillo, J. A. (2012). Diagnóstico del estilo de aprendizaje predominante basado en minería de datos y el modelo de Felder: aplicaciones al Elearnig 3.0. En F. Guerra et al (coordres.) Estilos de aprendizaje: investigaciones y experiencias, actas del V Congreso Mundial de Estilos de Aprendizaje, s.p.

Rendón-Lopez, L. M. \& Ortega-Carrillo, J. A. (2018). Evaluación de la calidad de programas de radio universitaria. Revista Mediterránea de Comunicación/Mediterranean Journal of Communication, 9(1), 151-175. DOI: https://www.doi.org/10.14198/MEDCOM2018.9.1.10

Rendón-Lopez, L. M., Ortega-Carrillo, J. A. y Ortega-Maldonado, A. (2017). La formación del profesorado universitario para la producción en radios universitarias: El caso de la Escuela Iberoamericana de Radio Digital. En A. Gutierrez, A. García y R. Collado (Ed). Educación Mediática y Competencia Digital. Segovia: Universidad de Valladolid.

Rojano-Caceres, J. R., Morales-Luna, C., Rebolledo-Mendez, G,.Ortega-Carrillo, J.A., Muñoz-Arteaga, J.(2016) et al. Raise awareness in society about deafness: A proposal with Learning Objects and Scenarios. Procedia: Social and Behavioral Sciences, (228), 575-581. http://www.headconf.org/wp-content/uploads/pdfs/2840.pdf

Russell, J. A. (1980). A circumplex model of affect. Journal of Personality and Social Psychology, 39(6), 1161-1178. http://doi.org/10.1037/h0077714

Shen, L., Callaghan, V. \& Shen, R. (2008) Afective e-Learning in residential and pervasive computing environments. Inf Syst Front 10: 461-472. DOI: 10.1007/s10796-0089104-5.

Shen, L. P., Leon, E., Callaghan, V., \& Shen, R. M. (2007). Exploratory Research on an Affective e-Learning Model. In J. Fong \& F. L. Wang (Eds.), Proceedings of Workshop on Blended Learning' 2007, 267-278. Recuperado de: https://dces.essex.ac.uk/staff/ vic/papers/2007_BL07(ExploratoryResearchOn).pdf

Shen, L., Wang, M., \& Shen, R. (2009). Affective e-Learning: Using "Emotional". Data to Improve Learning in Pervasive Learning Environment. Educational Technology \& Society, 12(2), 176-189. 
